\title{
Resultados del tratamiento multidisciplinario en cáncer de laringe. Estudio retrospectivo con seguimiento a 10 años
}

\author{
Outcome of multidisciplinary treatment in laryngeal cancer. Retrospective study with \\ 10-years follow-up
}

\begin{abstract}
Héctor Gurrola-Machuca ${ }^{*}$, Arturo P. Miranda-Aguirre', Michelle Villavicencio-Quejeiro², Gabriela Nuñez-Guardado² y Alejandro Juárez-Ramiro ${ }^{3}$

${ }^{1}$ Servicio de Cirugía Oncológica; ${ }^{2}$ Servicio de Radioterapia; ${ }^{3}$ Servicio de Oncología Médica. Clínica Multidisciplinaria de Tumores de Cabeza y Cuello, Centro Médico Nacional 20 de Noviembre, Instituto de Seguridad y Servicios Sociales de los Trabajadores del Estado, Ciudad de México,
\end{abstract} México

\section{Resumen}

Antecedentes: En cáncer de laringe, el tratamiento multidisciplinario mejora la calidad de vida del paciente y la posibilidad de preservar la laringe. La mayor parte de estos cánceres se presentan localmente avanzados. El objetivo es presentar los resultados de acuerdo con la etapa. Método: Estudio retrospectivo en el que se analizaron la etapa clínica, el tipo de tratamiento primario, los resultados y la sobrevida. Resultados: Se incluyeron 451 pacientes. La mediana de edad fue de 66 años. El mayor porcentaje de los tumores se presentó en etapa avanzada (72\%) y el sitio más afectado fue la glotis (84.5\%). En etapa temprana, el tratamiento más frecuente fue la radioterapia. En las etapas III y IVA, el 65\% fueron operables. En la etapa IVB el manejo fue no quirúrgico, con control en el $26 \%$ de los casos. La supervivencias a 10 años se relacionaron con la etapa clínica: $81.7 \%$ para la etapa I y 0\% para las etapas IVB y IVC. Conclusiones: Los pacientes con cáncer de laringe deben ser tratados de acuerdo con la etapa clínica y mediante un abordaje multidisciplinario. El seguimiento a largo plazo demostró un peor pronóstico para las etapas clínicas avanzadas.

Palabras Clave: Neoplasias de laringe. Laringectomía. Quimioterapia de inducción. Tratamiento concomitante. Biorradioterapia. Neoplasias de cabeza y cuello.

\begin{abstract}
Background: In laryngeal cancer, multidisciplinary treatment improves the patient's quality of life and the possibility of preserving the larynx. Most cases occur in a locally advanced stage. The aim is to present the results according to the stage. Method: A retrospective study which analyzed the clinical stage, type of primary treatment, outcomes, and survival were analyzed. Results: 451 patients were included. The median age was 66 years. The majority of the tumors presented in advanced stage (72\%) and the most affected subsite was the glottis (84.5\%). In the early stage the most frequent treatment was radiotherapy as the only treatment modality. In stages III and IVA, 65\% were resectable. In stage IVB the management was non-surgical, with control in $26 \%$ of the cases. Survival at 10 years was related to the clinical stage: $81.7 \%$ for stage I
\end{abstract}

\footnotetext{
Correspondencia:

*Héctor Gurrola-Machuca

Querétaro, 147-705

Col. Roma, Alcaldía Cuauhtémoc

Fecha de aceptación: 30-08-2019

Cir Cir. 2020;88(4):-466

C.P. 06700, Ciudad de México, México

Fecha de recepción: 09-12-2019

DOI: $10.24875 / C I R U .19001540 \quad$ www.cirugiaycirujanos.com

Contents available at PubMed

E-mail: gurrolamahprodigy.net.mx

0009-7411/@ 2019 Academia Mexicana de Cirugía. Publicado por Permanyer. Este es un artículo open access bajo la licencia CC BY-NC-ND (http://creativecommons.org/licenses/by-nc-nd/4.0/).
} 
and $0 \%$ for stages IVB and IVC. Conclusions: Patients with laryngeal cancer should be treated according to the clinical stage, through a multidisciplinary approach. Long-term follow-up showed a worse prognosis for advanced clinical stages.

Key Words: Larynx neoplasm. Laryngectomy. Induction chemotherapy. Chemoradiotherapy. Bioradiotherapy. Head and neck neoplasm.

\section{Introducción}

El carcinoma epidermoide de cabeza y cuello representa el $6 \%$ de todas las neoplasias, tanto en el ámbito mundial como en el nacional. El cáncer de laringe es la segunda neoplasia más común de la vía aerodigestiva alta y representa el $2 \%$ de todo el cáncer en el mundo; en nuestro país se ha estimado alrededor del $1 \%{ }^{1}$. El carcinoma epidermoide es la histología más frecuente, con un $98.7 \%$ de los casos ${ }^{2}$. El tratamiento del cáncer de laringe ha cambiado de manera significativa en los últimos años debido al desarrollo de los procedimientos de conservación de la laringe, los métodos de radioterapia y la introducción de la quimioterapia (inducción y concomitante), así como la adición de nuevos medicamentos como la terapia dirigida al blanco ${ }^{3}$. El manejo multidisciplinario mediante un equipo oncológico coordinado mejora la atención del paciente, su calidad de vida y la posibilidad de preservar la laringe ${ }^{4,5}$. Los factores de riesgo más involucrados han sido el alcohol, el tabaco y la combinación de ambos ${ }^{6}$. Se ha incrementado la evidencia de que algunos virus están implicados en la génesis del carcinoma epidermoide de cabeza y cuello, especialmente el virus del papiloma humano, el cual está presente en un 20 - 30\% de los casos de cáncer de laringe $\mathrm{e}^{7,8}$. La mayor parte de los casos (hasta el $70 \%$ en México) se encuentran en etapa localmente avanzada, es decir, III a IVB ${ }^{9}$.

El objetivo del presente estudio es reportar los resultados oncológicos de pacientes con cáncer de laringe, estratificados por etapa y tratados de forma multidisciplinaria en un centro de tercer nivel de atención, con un periodo largo de seguimiento.

\section{Método}

Se realizó un estudio retrospectivo y retrolectivo, en el cual se revisaron los expedientes de pacientes con diagnóstico de cáncer de laringe en un centro de tercer nivel de atención, que cumplieron con los siguientes criterios: mayores de 18 años, con diagnóstico histológico confirmado de carcinoma epidermoide de laringe, con expedientes completos y que recibieron todo el tratamiento en nuestro centro hospitalario. Se aseguró la confidencialidad de los sujetos a través de la codificación por sujeto, evitando el uso de nombres propios. Se contó con la aprobación del comité de ética en investigación de nuestra en institución. Se estudiaron los datos demográficos, como edad, sexo, subsitio, estado funcional por la escala del Eastern Cooperative Oncology Group (ECOG), etapa clínica según la clasificación del American Joint Committee on Cancer (AJCC) en su octava edición, tratamiento utilizado y sobrevida global. La sobrevida se analizó con la prueba de Kaplan-Meier y se compararon los factores con la prueba de log rank para demostrar diferencias significativas. Se consideró diferencia significativa un valor de $p<0.05$.

\section{Resultados}

Se incluyeron para su análisis 451 pacientes (el $85 \%$ de sexo masculino) con una mediana de edad de 66 años (rango de 33 a 94 años). El subsitio más afectado fue la glotis, en el $84.5 \%$ de los casos, seguido de la supraglotis y la hipofaringe, con un $13.5 \%$ y un $2 \%$, respectivamente. Los pacientes se presentaron en su mayoría con un ECOG de 1 y 2 (88.2\%). La etapa clínica temprana representó el $28 \%$ y las etapas localmente avanzadas el 72\% (Tabla 1).

- Etapa 0: se reportaron ocho casos, de los cuales siete se trataron con radioterapia externa por ser enfermedad multicéntrica y uno con denudación cordal. En todos los casos se controló la enfermedad.

- Etapas I y II: constituyeron 118 casos, de los cuales 82 estaban en etapa I y 36 en etapa II. De estos, 113 se trataron con radioterapia $(95.8 \%)$, cuatro con cordectomía (3.4\%) y uno con hemilaringectomía vertical y radioterapia posoperatoria (el cual no se controló). Se obtuvo control local en 117 casos, de los que 27 presentaron recurrencia (22.9\%). De estos últimos, en 23 se realizó cirugía con intento curativo, con lo que se obtuvo control local en 18 (78\%); la cirugía en 17 de ellos fue laringectomía radical y solo en uno se realizó hemilaringectomía vertical. 
Tabla 1. Datos clínicos y demográficos de los pacientes con cáncer de laringe

\begin{tabular}{lc}
\hline Variable & $\mathbf{n}=\mathbf{4 5 1}$ \\
\hline Edad, mediana (rangos) & $66(33-94)$ \\
Sexo, hombres (\%) & $384(85.1)$ \\
Subsitio, n (\%) & \\
Glotis & $381(84.5 \%)$ \\
Supraglotis & $61(13.5)$ \\
Hipofaringe & $9(2.0)$ \\
ECOG, n (\%) & \\
O & \\
I & $41(9.1)$ \\
II & $324(71.8)$ \\
III & $74(16.4)$ \\
IV & $11(2.4)$ \\
Etapa clínica, n (\%) & $1(0.2)$ \\
O & \\
I & $8(1.8)$ \\
II & $82(18.2)$ \\
III & $36(8)$ \\
IVA & $108(23.9)$ \\
IVB & $186(41.2)$ \\
IVC & $28(6.2)$ \\
ECOG: Eastern Cooperative Oncology Group. & $3(0.7)$ \\
\hline
\end{tabular}

En contraste, en las etapas localmente avanzadas, es decir, las etapas clínicas III y IVA, el $65.1 \%$, fueron operables. La glotis fue el subsitio mas afectado, en el $84.5 \%$ de los casos. Se presentaron 90 recurrencias y solo en 23 pacientes (25.5\%) se pudo controlar la enfermedad con cirugía curativa. Se desglosan los resultados a continuación:

- Etapa III: fueron 108 casos y solo se trataron 102, ya que seis pacientes no aceptaron ningún tratamiento. Todos los tratamientos tuvieron un abordaje multidisciplinario. Los tratamientos con los que se obtuvó mayor sobrevida fueron cetuximab y radioterapia concomitante, quimioterapia de inducción con manejo concomitante con cetuximab-radioterapia, y quimioterapia, cetuximab y radioterapia concomitante. La laringectomía radical con radioterapia posoperatoria, la laringectomía radical sola y la quimio-radioterapia (QT-RT) secuencial obtuvieron menores tasas de sobrevida que los primeros esquemas mencionados (Fig. 1A). Se obtuvo el control de la enfermedad en el $80 \%$ de los casos. Presentaron recurrencia 22 pacientes $(27 \%)$, de los cuales solo se pudo efectuar cirugía de curativa en dos (9\%). La quimioterapia de inducción mostró un aumento de la supervivencia (media: 8.23 años; intervalo de confianza del 95\% [IC 95\%], 5.92-10.5) en comparación con los que no la recibieron (media: 2.42 meses; IC 95\%, 2.14 - 2.7) (Fig. 1B). Para su análisis se categorizaron los tratamientos en tres grupos de pacientes: los que recibieron biorradioterapia, los que recibieron cirugía y los que recibieron QT-RT. Observamos que cuando se añadió cetuximab a la radioterapia para el manejo locorregional la supervivencia fue mayor que con cirugía sola o con QT-RT concomitante sin el uso de este anticuerpo (Fig. 1C).

- Etapa IVA: fueron 186 casos y se trataron 165 pacientes. El manejo fue multidisciplinario e incluyó 24 combinaciones de tratamientos diferentes. Los de mayor supervivencia fueron quimioterapia de inducción y concomitancia con radioterapia y cetuximab, seguido por los tratados con cirugía, disección radical de cuello y radioterapia, y en tercer lugar los correspondientes a laringectomía radical (Fig. 2A). Se obtuvo el control de la enfermedad en el $56 \%$ de los casos, de los cuales presentaron recurrencias 38 pacientes $(41 \%)$, y solo se pudo efectuar cirugía curativa en tres pacientes (8\%). El uso de quimioterapia de inducción mejoró la supervivencia (Fig. 2B). En relación con el manejo locorregional, ya sea concomitante, cirugía o radioterapia sola, la supervivencia es la misma (Fig. 2C).

- Etapa IVB: fueron 28 casos y solo se trataron 23 pacientes con 17 estrategias distintas. Los mejores resultados se obtuvieron con quimioterapia de inducción y QT-RT concomitante, o QT/RT secuencial. Se controlaron seis pacientes (26\%), recurrieron tres y ninguno se pudo rescatar.

- Etapa IVC: fueron tres pacientes y solo se trataron dos; ninguno se pudo controlar.

La mediana (rangos) de seguimiento para la etapa 0 fue de 8.39 años (1 - 21 años), para la etapa I fue de 6.15 años (0 - 28 años), para la etapa II fue de 6.34 años (0 a 36 años), para la etapa III fue de 2.67 años (0 - 31 años), para la etapa IVA fue de 1.51 años (0 - 17 años), para la etapa IVB fue de 1.08 años (0 - 9 años) y para la etapa IVC fue de 2.88 años ( 0 - 6 años).

La supervivencias por etapa a los 5 y 10 años fueron las siguientes: para la etapa 0 , del $100 \%$ en ambos periodos de tiempo; para la etapa I, del $86.3 \%$ y el $81.7 \%$; para la etapa II, del $81.2 \%$ y el $75.8 \%$; para la etapa III, del $46.5 \%$ y el $41.3 \%$; para la etapa IVA del $28.3 \%$ y el $24.9 \%$; para la etapa IVB, del $8.7 \%$ y el $0 \%$; y para la etapa IVC, solo un paciente sobrevivía a los 5 años (Fig. 3). 
A

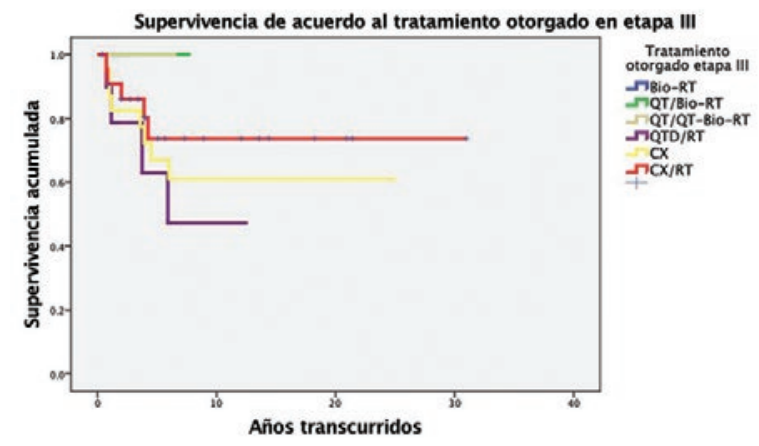

B

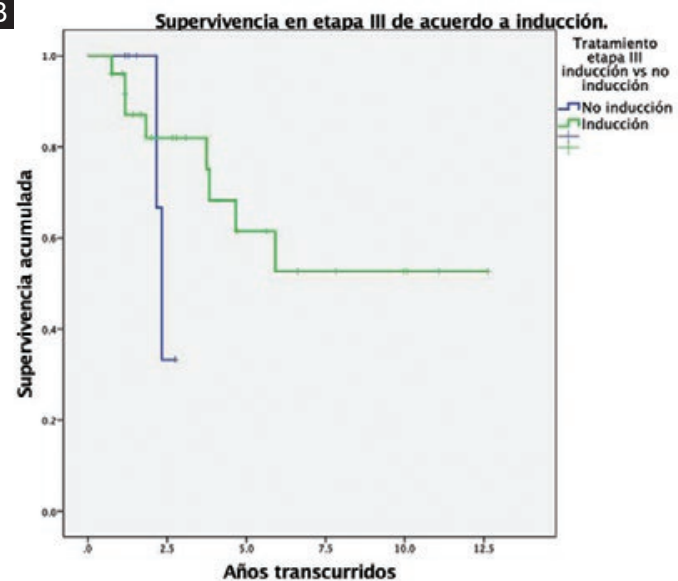

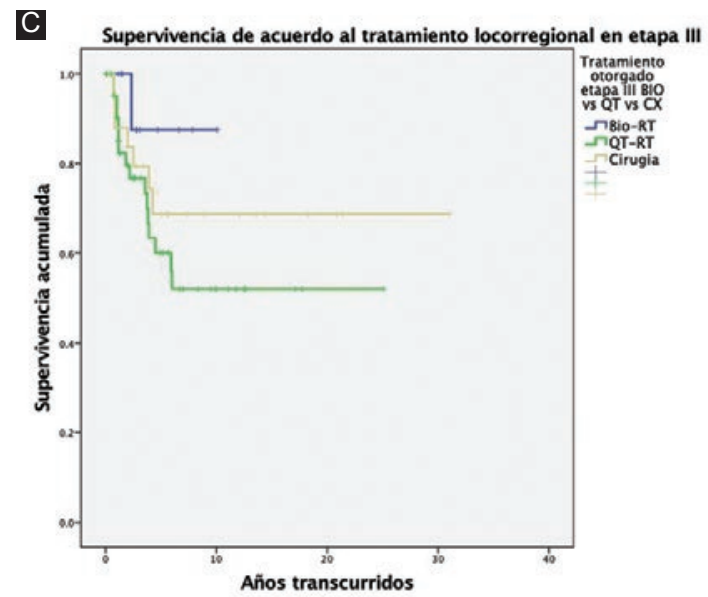

Figura 1. Supervivencia para las etapas III. A: supervivencia de acuerdo al tratamiento otorgado con las modadlidades. B: supervivencia de acuerdo al uso de quimioterapia de inducción. C: supervivencia de acuerdo al uso de biorradioterapia. Bio: cetuximab; CX: cirugía; QT: quimioterapia; QTD: doblete; $R T$ : radioterapia.

\section{Discusión}

En nuestra población, la edad, el sexo y el subsitio de presentación fueron semejantes a los reportados en la literatura mundial ${ }^{10}$.

El tratamiento del cáncer de laringe depende de la etapa clínica (temprana o avanzada), de los resultados funcionales esperados, del deseo del paciente y de la experiencia del equipo multidisciplinario en el manejo del cáncer de laringe ${ }^{4}$.

En las etapas tempranas $0, \mathrm{I}$ y II, el tratamiento principal en nuestro estudio fue la radioterapia externa, ya que se obtiene el mismo control que con cirugía y los resultados de la función de la voz son mejores $^{11,12}$. En caso de recurrencia se ofrece cirugía de rescate, con un control de la enfermedad muy alto ${ }^{13}$.

En la etapa III, la cirugía ya no es la primera opción de tratamiento, pues ha sido sustituida por la preservación de órgano con radioterapia concomitante, ya sea con cetuximab o sales platinadas ${ }^{14,15}$. Otras opciones de manejo son la quimioterapia de inducción en pacientes con gran carga tumoral ${ }^{16}$, o bien la cirugía sola si el paciente la acepta, con o sin radioterapia adyuvante $^{17,18}$. En presencia de recurrencia es posible efectuar cirugía curativa en pocos casos $(9 \%)^{19,20}$.

En la etapa IVA está indicada la cirugía ${ }^{21,22}$. Sin embargo, en los pacientes que la rechazan se podría considerar el tratamiento con quimioterapia de inducción ${ }^{16}$. Si estos presentan una respuesta mayor del $50 \%$, nuestra recomendación es continuar con manejo concomitante con cetuximab o sales platinadas ${ }^{15}$. En caso de no obtener esta respuesta, serían candidatos a laringectomía radical ${ }^{23,24}$. Ningún tratamiento locorregional es superior a los demás.

Los pacientes en etapa IVB, debido a la gran carga tumoral que presentan, deben ser tratados con quimioterapia de inducción y posterior manejo concomitante con cetuximab o quimioterapia, según su estado funcional ${ }^{25}$. El pronóstico en general es malo a corto plazo. 

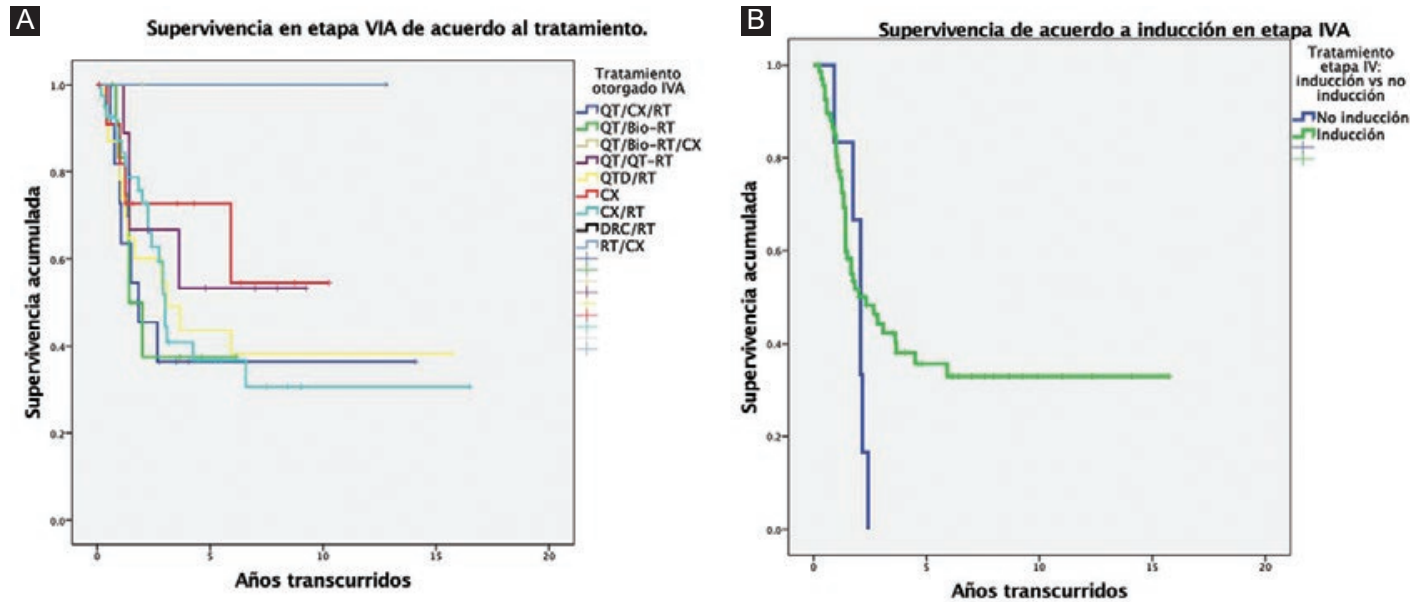

C Supervivencia de acuerdo al tratamiento locorregional en
etapa IVA

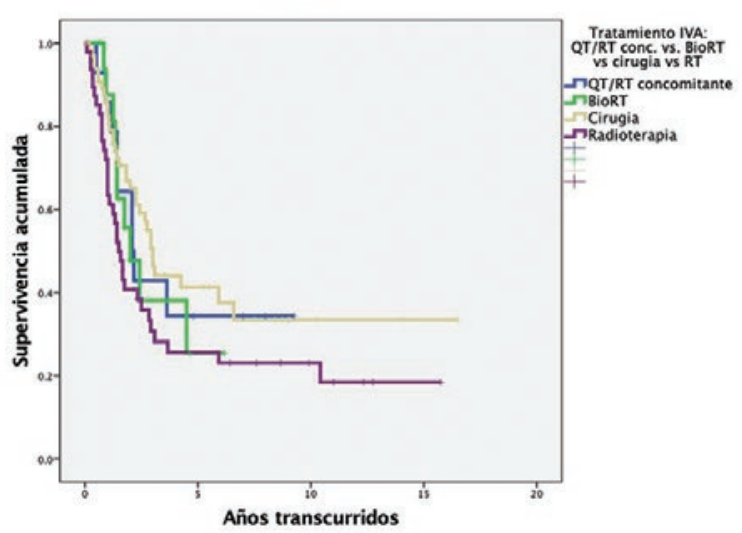

Figura 2. Supervivencia para las etapas IV. A: supervivencia al tratamiento otorgado con las nueve modalidades. B: supervivencia de acuerdo al uso de quimioterapia de inducción. C: supervivencia de acuerdo a la estrategia de tratamiento locorregional. Bio: cetuximab; CX: cirugía; DRC: disección radical de cuello; QT: quimioterapia; QTD: doblete; $R T$ : radioterapia.

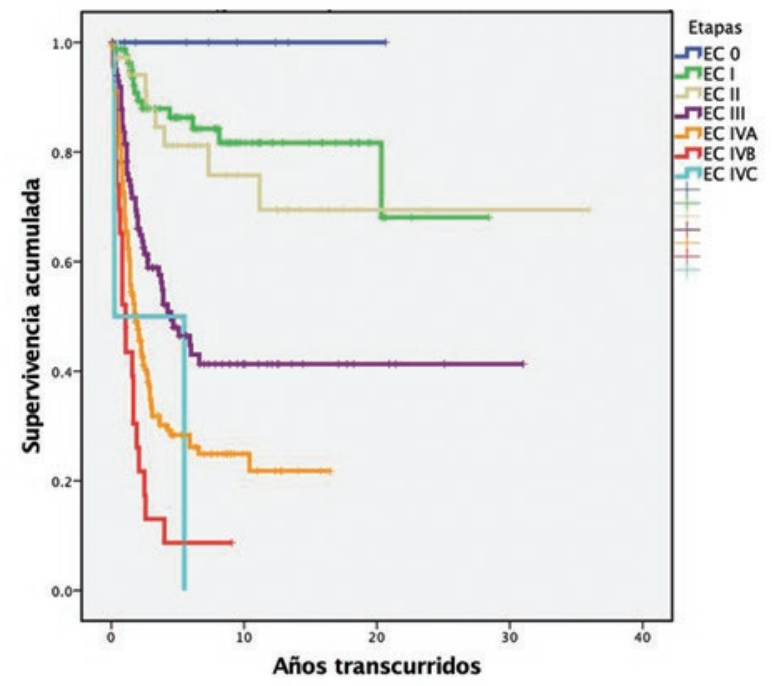

Figura 3. Supervivencia según la etapa.

En nuestro estudio solo hubo dos pacientes en etapa IVC, mismos que recibieron quimioterapia de inducción sin respuesta. Esto se otorgó previo a contar con los resultados del estudio EXTREME ${ }^{26}$, que probablemente hubieran cambiado el esquema de tratamiento que se les ofreció.

\section{Conclusiones}

Los pacientes con cáncer de laringe deben ser tratados de acuerdo con la etapa clínica, su comorbilidad y la experiencia del equipo multidisciplinario especializado en cáncer de cabeza y cuello.

En la etapa clínica 0 , si la lesión es localizada debe ser tratada con denudación cordal, o bien con radioterapia externa en pacientes con tumores multicéntricos o recurrentes. En las etapas I y II, el tratamiento con mejores resultados funcionales y calidad de la voz es la radioterapia externa, aunque también hay opciones quirúrgicas. En la etapa clínica III, el objetivo es la preservacion del órgano, por lo que el tratamiento debe ser el manejo concomitante con cetuximab o quimioterapia. La quimioterapia de inducción podría 
aumentar la supervivencia en esta etapa. En la etapa clínica IVA está indicada la cirugía, aunque esta no aumenta la supervivencia en comparación con un manejo concomitante. En estos pacientes, aparentemente la quimioterapia de inducción aumenta la supervivencia. Por su carga tumoral, las etapas IVB deben ser tratadas con quimioterapia de inducción o manejo concomitante cetuximab o quimioterapia, de acuerdo a su estado funcional y comorbilidades. Las Etapa IVC, deben ser tratados con quimioterapia.

Este estudio presenta la limitación de ser retrospectivo, por lo que se deberán evaluar los resultados oncológicos en estudios prospectivos, con los lineamientos de tratamiento propuestos y con un seguimiento a largo plazo.

\section{Agradecimientos}

A los pacientes que confiaron en nosotros.

\section{Conflicto de intereses}

Los autores declaran que no existió conflicto de intereses durante el desarrollo y la redacción de este escrito.

\section{Responsabilidades éticas}

Protección de personas y animales. Los autores declaran que para esta investigación no se han realizado experimentos en seres humanos ni en animales.

Confidencialidad de los datos. Los autores declaran que han seguido los protocolos de su centro de trabajo sobre la publicación de datos de pacientes.

Derecho a la privacidad y consentimiento informado. Los autores han obtenido el consentimiento informado de los pacientes y/o sujetos referidos en el artículo. Este documento obra en poder del autor de correspondencia.

\section{Bibliografía}

1. Global Cancer Observatory. (Consultado el 2/5/2019.) Disponible en: http://gco.iarc.fr

2. Shen Z, Lin L, Cao B, Zhou C, Hao W, Ye D. LZTS2 promoter hypermethylation: a potential biomarker for the diagnosis and prognosis of laryngeal squamous cell carcinoma. World J Surg Oncol. 2018;16:42.

3. Steuer CE, El-Deiry M, Parks JR, Higgins KA, Saba NF. An update on larynx cancer. CA Cancer J Clin. 2017;67:31-50.
4. Obid R, Redlich $\mathrm{M}$, Tomeh $\mathrm{C}$. The treatment of laryngeal cancer. Oral Maxillofac Surg Clin N Am. 2019;31:1-11.

5. Rosa V, Fores J, Silva E, et al. Interdisciplinary interventions in the perioperative rehabilitation of total laryngectomy: an integrative review. Clinics. 2018:73(Suppl 1). doi:10.6061/clinics/2018/e484s

6. Salvador-Coloma C, Cohen E. Multidisciplinary Care of Laryngeal Cancer. J Oncol Pract. 2016;12(8):717-724. doi:10.1200/JOP.2016.014225

7. Gama RR, Carvalho AL, Longatto Filho A, Scorsato AP, López RV, Rautava J, et al. Detection of human papillomavirus in laryngeal squamous cell carcinoma: Systematic review and meta-analysis. Laryngoscope. 2016;126:885-93.

8. Li X, Gao L, Li H, Gao J, Yang Y, Zhou F, et al. Human papillomavirus infection and laryngeal cancer risk: a systematic review and meta-analysis. J Infect Dis. 2013;207:479-88.

9. Herrera-Gómez A. Demografía del cáncer laríngeo en el Instituto Nacional de Cancerología. Cir Cir. 2009;77:353-7.

10. Morse E, Fujiwara RJT, Judson B, Mehra S. Treatment delays in laryngeal squamous cell carcinoma: a national cancer database analysis. Laryngoscope. 2018;128:2751-8

11. van Loon Y, Hendriksma M, Langeveld TPM, de Jong MA, Baatenburg de Jong RJ, Sjögren EV. Treatment preferences in patients with early glottic cancer. Ann Otol Rhinol Laryngol. 2018;127:139-45.

12. Patel KB, Nichols AC, Fung K, Yoo J, MacNeil SD. Treatment of early stage supraglottic squamous cell carcinoma: meta-analysis comparing primary surgery versus primary radiotherapy. Otolaryngol Head Neck Surg. 2018;47:19

13. Cheraghlou S, Kuo P, Mehra S, Yarbrough WG, Judson BL. Salvage surgery after radiation failure in T1/T2 larynx cancer: outcomes following total versus conservation surgery. Otolaryngol Head Neck Surg. 2018; 158:497-504.

14. Tang Z-X, Gong J-L, Wang Y-H, Li ZH, He Y, Liu YX, et al. Efficacy comparison between primary total laryngectomy and nonsurgical organ-preservation strategies in treatment of advanced stage laryngeal cancer: A meta-analysis. Medicine (Baltimore). 2018;97:e10625.

15. Mesía R, García-Sáenz JA, Lozano A, Pastor M, Grau JJ, Martínez-Trufero $\mathrm{J}$, et al. Could the addition of cetuximab to conventional radiation therapy improve organ preservation in those patients with locally advanced larynx cancer who respond to induction chemotherapy? An Organ Preservation Spanish Head and Neck Cancer Cooperative Group Phase 2 Study. Int J Radiat Oncol Biol Phys. 2017;97:473-80.

16. Lefebvre JL, Pointreau Y, Rolland F, Alfonsi M, Baudoux A, Sire C, et al. Induction chemotherapy followed by either chemoradiotherapy or bioradiotherapy for larynx preservation: the TREMPLIN randomized phase II study. J Clin Oncol. 2013;31:853-9.

17. Smee R, Williams JR, Kotevski DP. Management of locally advanced T3-4 glottic laryngeal carcinomas. J Laryngol Otol. 2018;132:642-50.

18. Li M, Zhang T, Tan B, Yu M, Zhang B. Role of postoperative adjuvant radiotherapy for locally advanced laryngeal cancer: a meta-analysis. Acta Otolaryngol (Stockh). 2019;139:172-7.

19. Hay A, Simo R, Hall G, Tharavai S, Oakley R, Fry A, et al. Outcomes of salvage surgery for the oropharynx and larynx: a contemporary experience in a UK Cancer Centre. Eur Arch Otorhinolaryngol. 2019;276:1153-9.

20. Pantvaidya GH, Raina S, Mondal A, Deshmukh A, Nair D, Pai $P$, et al. Total laryngectomy: surgical morbidity and outcomes - a case series. Indian J Cancer. 2017;54:621-5.

21. Francis E, Matar N, Khoueir N, Nassif C, Farah C, Haddad A. T4a laryngeal cancer survival: retrospective institutional analysis and systematic review. Laryngoscope. 2014;124:1618-23

22. García Lorenzo J, Montoro Martínez V, Rigo Quera A, Codina Aroca A López Vilas M, Quer Agustí M, et al. Modifications in the treatment of advanced laryngeal cancer throughout the last 30 years. Eur Arch Otorhinolaryngol. 2017;274:3449-55.

23. Gorphe $\mathrm{P}$, Matias M, Blanchard P, Even C, Ferte C, Tao Y, et al. Outcomes following laryngectomy refusal after insufficient response to induction chemotherapy. Laryngoscope. 2017;127:1791-6.

24. Semrau S, Schmidt D, Lell M, Waldfahrer F, Lettmaier S, Kuwert T, et al. Results of chemoselection with short induction chemotherapy followed by chemoradiation or surgery in the treatment of functionally inoperable carcinomas of the pharynx and larynx. Oral Oncol. 2013;49:454-60.

25. Nishikawa D, Hanai N, Ozawa T, Hirakawa $H$, Suzuki $H$, Nakashima $T$, et al. Role of induction chemotherapy for N3 head and neck squamous cell carcinoma. Auris Nasus Larynx. 2015;42:150-5.

26. Vermorken JB, Mesia R, Rivera F, Remenar E, Kawecki A, Rottey S, et al. Platinum-based chemotherapy plus cetuximab in head and neck cancer. N Engl J Med. 2008;359:1116-27. 